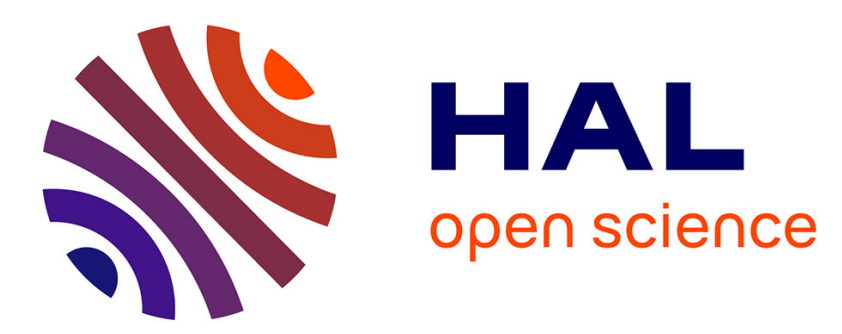

\title{
Performance Comparison of Digital Modulations in the Presence of Gaussian Phase Noise in the Sub-THz Context
}

Paul Desombre, Haïfa Farès, Yves Louët

\section{- To cite this version:}

Paul Desombre, Haïfa Farès, Yves Louët. Performance Comparison of Digital Modulations in the Presence of Gaussian Phase Noise in the Sub-THz Context. 2021 Fourth International Workshop on Mobile Terahertz Systems (IWMTS), Jul 2021, Essen, Germany. pp.1-5, 10.1109/IWMTS51331.2021.9486786 . hal-03323900

\section{HAL Id: hal-03323900 \\ https://hal.science/hal-03323900}

Submitted on 23 Aug 2021

HAL is a multi-disciplinary open access archive for the deposit and dissemination of scientific research documents, whether they are published or not. The documents may come from teaching and research institutions in France or abroad, or from public or private research centers.
L'archive ouverte pluridisciplinaire HAL, est destinée au dépôt et à la diffusion de documents scientifiques de niveau recherche, publiés ou non, émanant des établissements d'enseignement et de recherche français ou étrangers, des laboratoires publics ou privés. 


\title{
Performance Comparison of Digital Modulations in the Presence of Gaussian Phase Noise in the Sub-THz Context
}

\author{
Paul Desombre*, Haïfa Farès* and Yves Louët* \\ ${ }^{*}$ IETR/CentraleSupélec, Campus de Rennes - 35576 Cesson-sévigné, France \\ Email: name.surname@ centralesupelec.fr
}

\begin{abstract}
Driven by the need of more available frequency bands, an increasing attention has been paid to the use of subTHz bands. In such high frequency bands, the signal is expected to be impaired by phase noise, I/Q mismatch, power amplifier non linearities, etc. It has been shown that at such frequencies, with possibly large bandwidth ( $\sim 50 \mathrm{GHz})$, Gaussian phase noise models accurately the phase noise. This paper studies the effect of Gaussian phase noise on several waveforms. Here, five performance metrics are used to compare them: Bit Error Rate (BER), Peak-to-Average Power Ratio (PAPR), Adjacent Channel Power Ratio (ACPR), spectral efficiency (SE) and Error Vector Magnitude (EVM). The aim of this comparison is to give recommandations on which waveform to use in this context, depending on the impact of the phase noise on the performance of such transmissions.
\end{abstract} form

Index Terms-Sub-THz communications, Phase Noise, Wave-

\section{INTRODUCTION}

Considering the latest report of the European Communications Office (ECO) Frequency Information System (FIS) [1], frequencies over $71 \mathrm{GHz}$ have yet to be allocated to wideband data communications. In the last decades, the research community payed more attention to the sub- $\mathrm{THz}$ bands (200 $\mathrm{GHz}-1 \mathrm{THz}$ ) [2]. However, with higher frequencies comes several impairments, such as the Phase Noise (PN), which is the subject of this paper.

Phase noise is the main distorsion experienced by the signal in the sub- $\mathrm{THz}$ bands, which makes its recovery impossible. Therefore, its impact has been widely studied [3]-[7]. Indeed, in [3], Bicais et al. compared two models of PN: correlated Wiener plus Gaussian PN, and uncorrelated Gaussian PN (GPN). They showed that, for broadband communications, such as sub-THz communications, uncorrelated GPN is an accurate model. An other study of PN [4] gives a model for PN simulations, with a thourough statistical study with the goal of being more accurate than other models, at the cost of complexity. The authors from [5], [6] gave a design of digital communications for strong PN channels: their contributions showed that, by using polar metric in the detector, the performance in terms of Bit Error Rate (BER) is improved. An other digital modulation framework has been proposed in [8], where PN robustness has been considered as the performance metric to optimize.
In the review of the $\mathrm{THz}$ communications advances drawn in [2], the author explained that publications have been numerous since early 2000. Communication systems using all-electronics (such as [7]) or photonics are using carrier frequencies in the range $100 \mathrm{GHz}-700 \mathrm{GHz}$, though the most studied frequency bands lies between $200 \mathrm{GHz}$ and $400 \mathrm{GHz}$. From this review, researchers have reached over $100 \mathrm{Gbps}$. These systems used OOK, QPSK and 16-QAM modulation schemes.

The aim of this paper is to compare multiple waveforms in the presence of GPN using the following five performance metrics: Bit Error Rate (BER), Peak to Average Power Ratio (PAPR), Adjacent Channel Power Ratio (ACPR), Spectral Efficiency (SE) and Error Vector Magnitude (EVM). Some well-known Phase-Amplitude Modulations (PAM) modula-

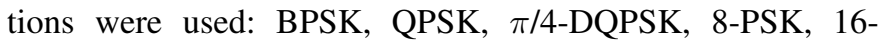
APSK, 16-QAM, 256-QAM and OOK. The OOK scheme was taken from the framework presented in [8]. Alongside widely used modulation schemes, Continuous Phase Modulation (CPM) have been considered into the comparison study due to their high energy efficiency. Another promising modulation scheme was used: Parallel Sequence Spread Spectrum (PSSS) [9] combined with BPSK and QPSK modulations. The goal of this comparison is to show the performance of different modulation schemes impaired by PN, with PN modelled as GPN. To the best of our knowledge, no comparison of such scale has been published so far.

The remainder of this paper is organized as follows. Section II briefly describes the channel and GPN models. Section III provides simulations scheme and results. Section IV illustrates the main outcomes of this comparison study. Finally, conclusions are drawn in Section V.

\section{SYSTEM MODEL}

In this work, we considered a SISO (Single-Input, SingleOutput) system with an Additive White Gaussian Noise (AWGN) channel including PN at both sides (transmitter and receiver). The received signal is defined as

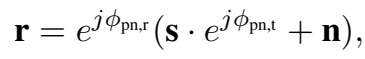

with $\mathbf{r}$ the received signal after the receiver's oscillator, $\mathbf{n}$ the circular, zero-mean, complex white Gaussian noise with variance $\sigma_{n}^{2}$, $\mathbf{s}$ the transmitted signal, and $\Phi_{\mathrm{pn}, t}$ and $\Phi_{\mathrm{pn}, r}$ the 
PN at the transmitter and the receiver side respectively. The transmitted signal is composed of symbols which take their values from the aforementioned modulations. Each of the two instances of PN follow an independant zero-mean Gaussian process $\phi_{\mathrm{pn}} \sim N\left(0, \sigma_{\mathrm{pn}}^{2}\right)$.

In our simulations, we used several modulation schemes: BPSK, QPSK, $\pi / 4$-DQPSK, 8-PSK, 16-APSK, 16-QAM, 256QAM, OOK, GMSK, PSSS ${ }_{B P S K}$ and PSSS ${ }_{Q P S K}$. To compute the PSSS, we need a spreading sequence (maximum length sequence), we used the first $2^{8}$-length sequence taken from [11]. All of our simulations were baseband, filtered by a Square-Root-Raised-Cosine (SRRC) filter with a roll-off factor of $\alpha=0.25$, except for the OOK modulation (NonReturn-to-Zero filter, NRZ) and GMSK (Gaussian filter, with parameters $\mathrm{L}=4$ and $\mathrm{BT}=0.25)$. The variance of the GPN was: $\sigma_{\mathrm{pn}}^{2}=10^{-4}$ for low PN, $\sigma_{\mathrm{pn}}^{2}=10^{-2}$ for medium PN and $\sigma_{\mathrm{pn}}^{2}=10^{-1}$ for high PN. No Forward Error Correction (FEC) nor equalization algorithms were used in our simulation setup.

The BER simulations used Monte-Carlo (MC) scheme, with $E_{b} / N_{0}=[0,1, \ldots, 30] \mathrm{dB}$, and frames of $2^{15}$ bits. To process the PAPR, we calculated the Complementary Cumulative Distribution Function (CCDF). The ACPR was obtained by looking at the normalized frequency of the first channel offset. In a normalized frequency setup, with the first channel offset taken at the normalized frequency value of $F_{\text {offset }}=0.16$. Spectral efficiency was investigated for a BER of $10^{-5}$. And EVM was processed at $E_{b} / N_{0}=[0,5,10,15,30] \mathrm{dB}$. As EVM for the GMSK is not trivial to obtain, we chose to calculate its Mean Quadratic Error (MQE), which is given by

$$
\mathrm{MQE}=\frac{1}{N} \sum_{i=1}^{N}\left(\phi_{s, \mathrm{t}}(i)-\phi_{s, \mathrm{r}}(i)\right)^{2},
$$

with $\Phi_{s, t}$ and $\Phi_{s, r}$ are the phases for the ith sample at the transmitter and the receiver side, respectively, for the $i^{\text {th }}$ sample. The EVM for the other modulations were obtained thanks to the MATLAB comm.EVM object, with reference signal normalization.

In the following section, results are illustrated for medium and high level of PN for which the impact on performance metrics could be observed.

\section{Simulations}

\section{A. Bit error rate}

Figure 1 shows the BER as a function of the $E_{b} / N_{0}(\mathrm{~dB})$ for the simulated systems. All of the 11 modulations are shown, without and with PN (medium level) to enable comparison. As expected, medium PN renders 256-QAM unrecoverable. As highlighted in [5], the effect of PN on the signal's BER is that it reaches an error floor. With medium PN, the error floor is reached at $10^{-2}$ for 256-QAM and a little over $10^{-8}$ for $16-$ APSK. Low-order modulation waveforms have their respective error floor lower than $10^{-8}$.

In a system with high PN, the BER curves are shown on Figure 2. Most of the modulations exhibit an error floor. At

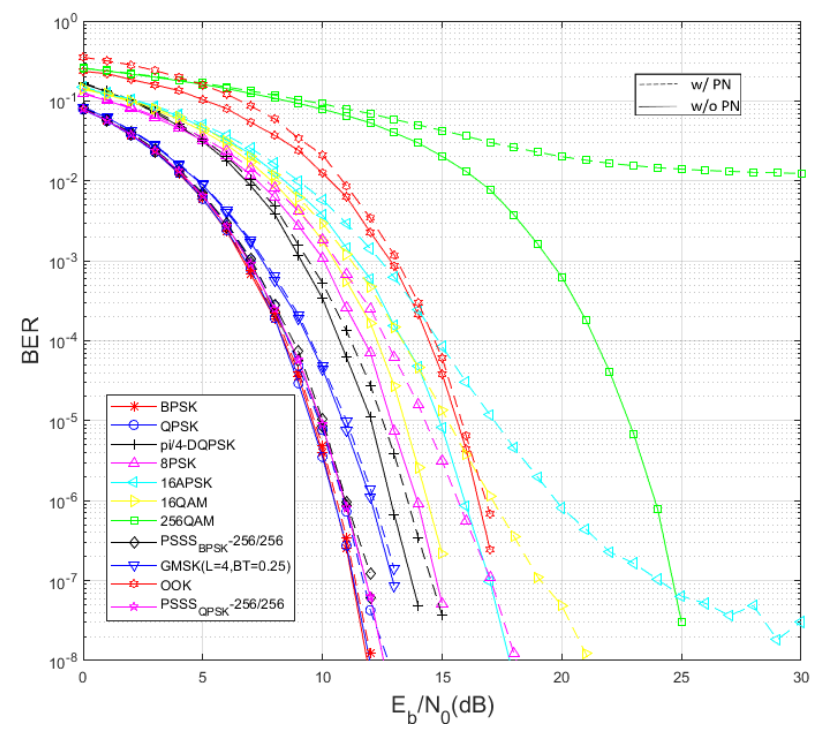

Fig. 1. BER curves for no PN and medium PN.

this point, only low-order modulations such as BPSK, QPSK, GMSK and the two PSSS remain recoverable. Though the QPSK scheme presents an error floor of $3 \cdot 10^{-7}$ at high $E_{b} / N_{o}$.

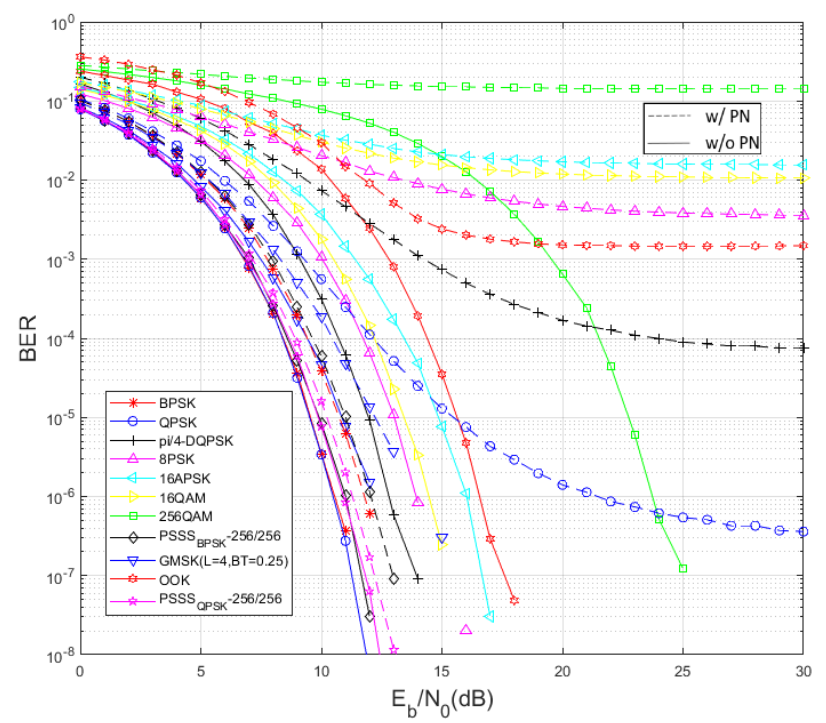

Fig. 2. BER curves for no PN and high PN.

Regarding only the BER performance, the modulations we should use in PN-impaired systems such as sub-THz communications should be low-order modulations. The PSSSmodulated BPSK and QPSK are remarkably robust to PN.

\section{B. Peak to Average Power Ratio}

If we look at the PAPRs represented by their CCDFs in Figure 3, the two PSSS are of the same order as Multi-Carrier waveforms. As shown in [10], in sub-THz systems, it is more preferable to use Single-Carrier (SC) systems. As expected, the GMSK is not illustrated in the CCDF Figure, as it has a null-PAPR. 


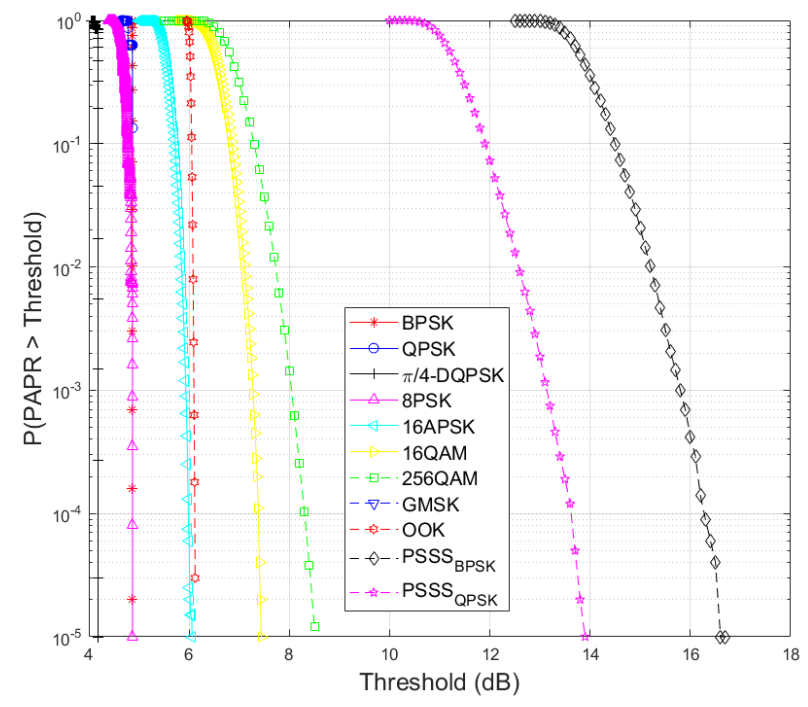

Fig. 3. PAPR CCDF of the simulated waveforms.

Regarding only the PAPR of the waveforms, the PSSS should be excluded of the possible waveforms for sub$\mathrm{THz}$ communications, whereas the other simulated waveforms could all be used.

\section{Adjacent Channel Power Ratio}

As the ACPR is processed on the Power Spectral Density (PSD) of the waveforms, it is expected that all the simulated waveforms using the same SRRC filter have exactly the same performance (Fig. 4). As the Gaussian filter used in the GMSK has slightly higher rejection than the SRRC in the vicinity of the main lobe, it is also not surprizing that the GMSK has better performance. Furthermore, as for the OOK, the use of an NRZ filter is causing spikes in its PSD, this explains its higher ACPR.

It should be noted that the PSD of a White Gaussian noise is constant. As the level of the PN PSD is higher than the level of the signal PSD in the rejection band, it explains the high increase of ACPR with the increase of PN.

Looking only at the ACPR, GMSK has the best behavior for these parameters. It is not excluded that, with a better filter, the other modulations would have better performance.

\section{Spectral efficiency}

Without PN (Fig. 5), SE is well known and should serve as a comparator for PN-impaired waveforms.

As seen earlier, the 256-QAM is excluded from the list of considered waveforms. Its BER does not reach the $10^{-5}$ threshold within the considered $E_{b} / N_{0}$ interval for medium PN (Fig. 6) and high PN (Fig. 7).

As for the other waveforms, with medium PN (Fig. 6), the $E_{b} / N_{0}$ level is higher than without PN, appart from the lower order modulations. This highlights the robustness to PN of low-order modulations.

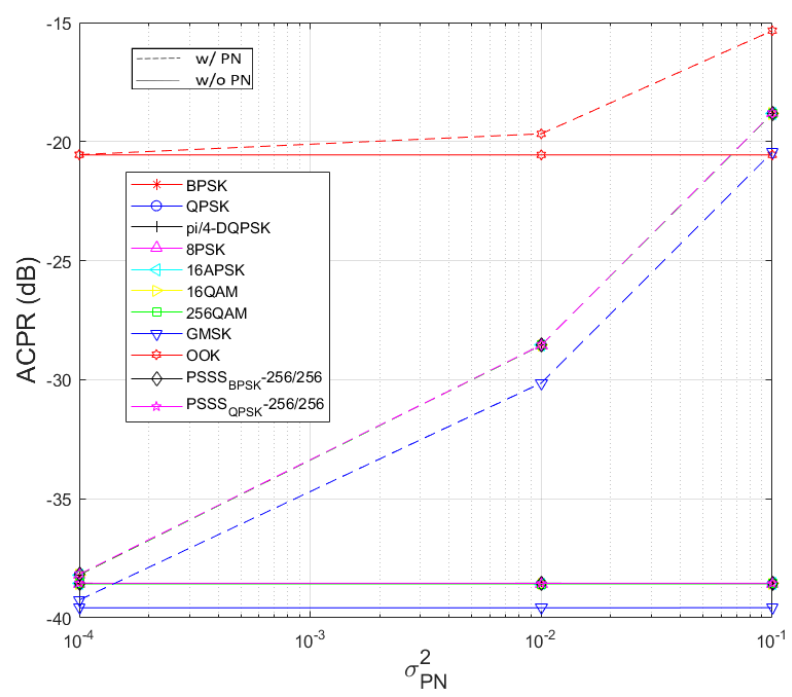

Fig. 4. ACPR of the simulated waveforms.

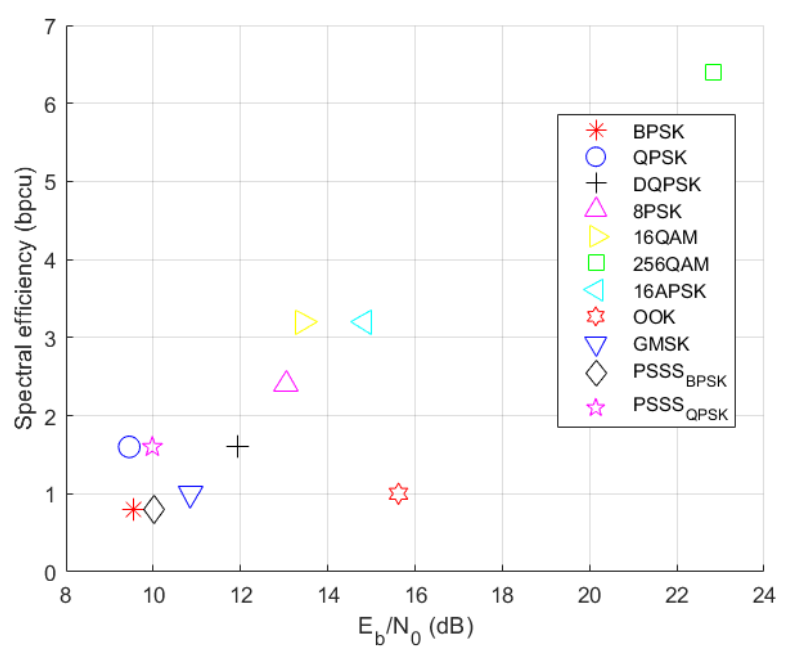

Fig. 5. SE performance without $\mathrm{PN}$ and $\mathrm{BER}=10^{-5}$.

As expected, with higher PN (Fig. 7), the same trend is observed, except for QPSK, which has as an $E_{b} / N_{0}$ of 15.33 $\mathrm{dB}$ for a BER of $10^{-5}$.

Then, if we tune on for an $E_{b} / N_{0}$ threshold of $16 \mathrm{~dB}$, which is the upper bound of $E_{b} / N_{0}$ for high PN, only the lower order modulations should be considered for a highly $\mathrm{PN}$-impaired system.

\section{E. Error vector magnitude}

At high $E_{b} / N_{0}$, the EVM is expected to be near $0 \%$, which can be observed in Figure 8. Without PN, for high order modulations, the EVM is indeed near $0 \%$. With low order modulations, the EVM is higher. The higher the $E_{b} / N_{0}$, the higher the increase of EVM in systems impaired by PN.

It can be said the same about MQE for GMSK modulation. However, the level of MQE is significantly lower for GMSK 


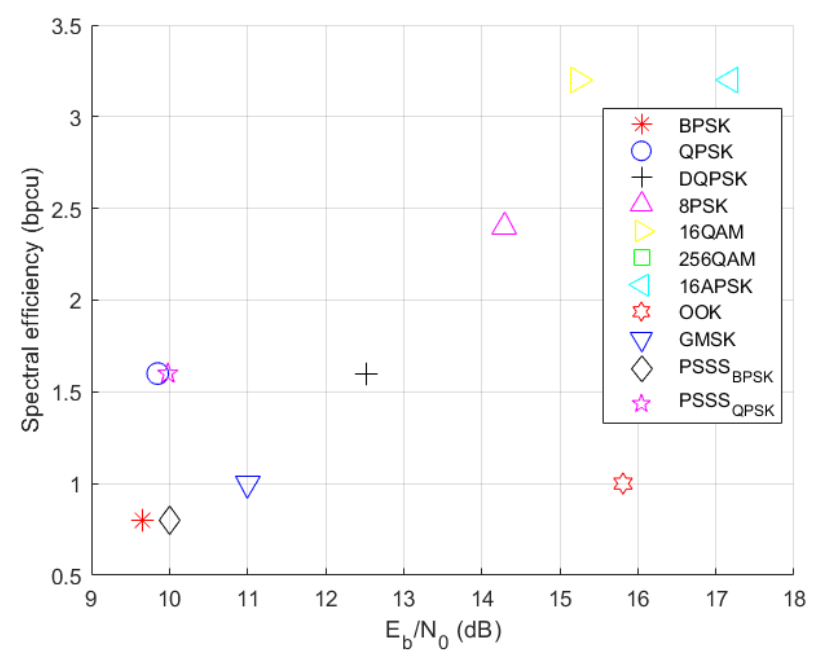

Fig. 6. SE performance with medium $\mathrm{PN}$ and $\mathrm{BER}=10^{-5}$.

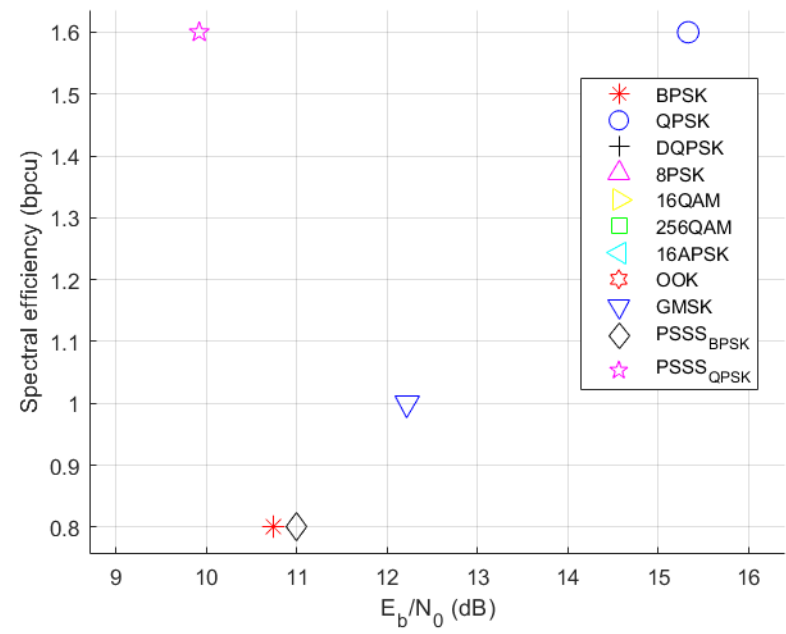

Fig. 7. SE performance with high $\mathrm{PN}$ and $\mathrm{BER}=10^{-5}$.

than the level of EVM for any low order modulation. This makes GMSK a good candidate for sub-THz communications.

Considering the EVM and MQE, GMSK is far ahead of the other waveforms.

\section{RESULTS AND DISCUSSION}

Now, instead of considering each criterion alone we consider them all together. To compare the waveforms, we graded objectively each of them on a scale from 0 to 10 , with 0 the worst candidate for sub- $\mathrm{THz}$ communications and 10 the best candidate (Fig. 10).

Given the results of the grading, in a system with little to no PN, depending on the channel conditions and other impairments, any modulation could be used. However, in a system impaired with medium to high PN, we advise to use waveforms with a low-order modulation, such as the QPSK or CPM such as GMSK.

QPSK has a relatively good robustness to PN, which is surely one of the reasons it is used in existing systems in

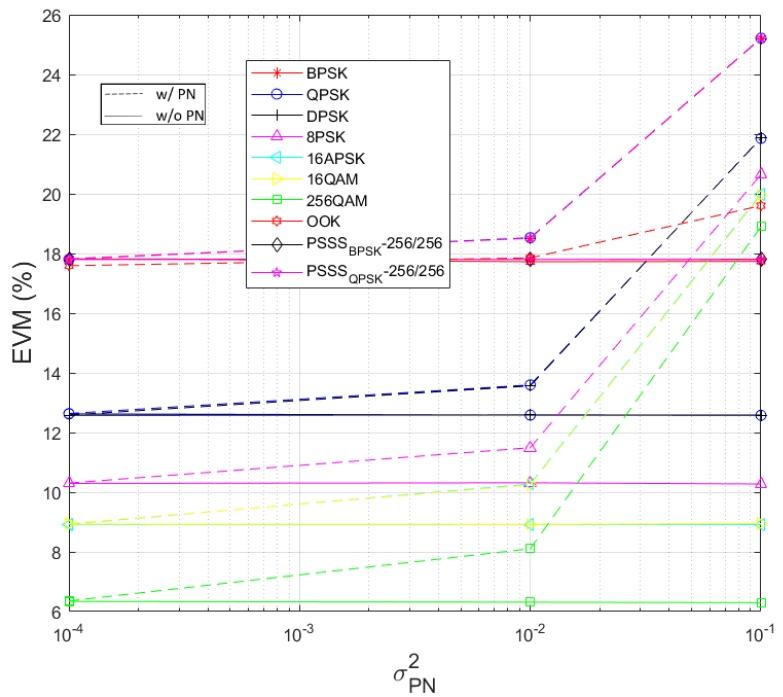

Fig. 8. EVM for $E_{b} / N_{o}=15 \mathrm{~dB}$.

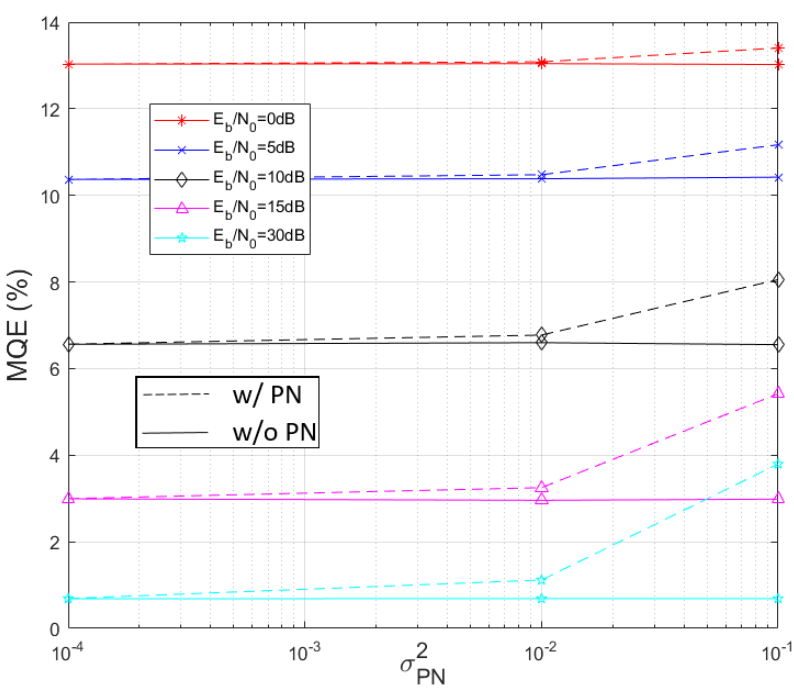

Fig. 9. MQE for GMSK modulation.

the literature [2]. QPSK also has objectively good EVM and PAPR performance. The ACPR depends on the shaping filter, which can be optimized. SE is low compared to higher order modulations, but with large bandwidth, even low SE can enable high throughput.

On the other hand, GMSK has better performance compared to the QPSK in presence of high PN. It also has the best PAPR possible (since it has a constant complex envelope). Its MQE is excellent, and it has the best ACPR among the studied waveforms. Nevertheless, its complexity depends on the number of states in the Viterbi algorithm needed to perform Maximum Likelihood detection. Therefore, its complexity is obviously higher than that of the QPSK scheme, based on a simple separator. And yet this GMSK remains of great interest thanks to its high energy efficiency. Indeed, Since GMSK has a constant complex envelope, it would enable the use of Power 


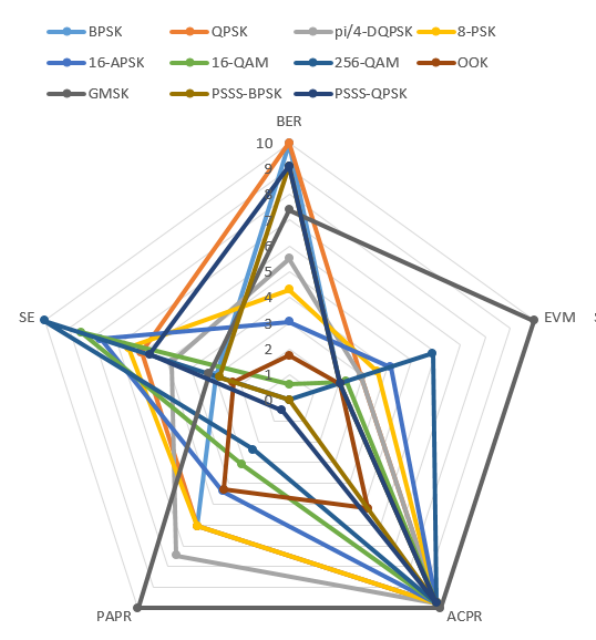

(a)

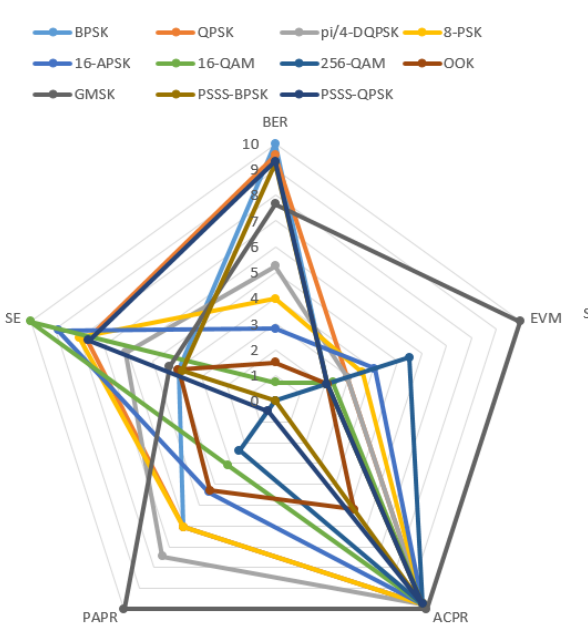

(b)

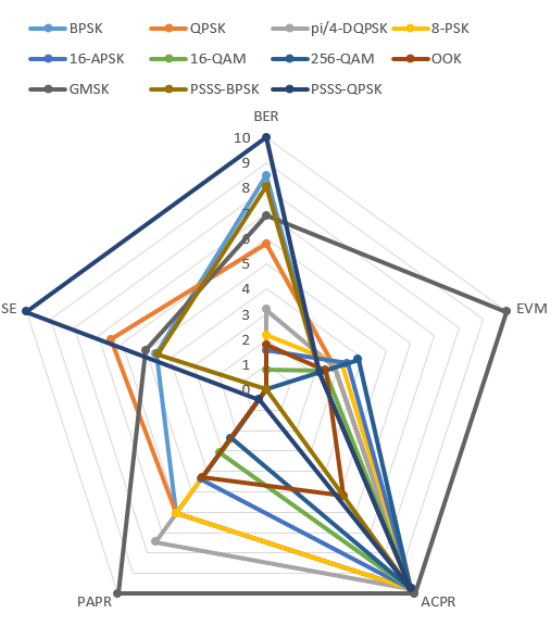

(c)

Fig. 10. Comparison of each waveform with (a) no phase noise, (b) medium phase noise, (c) high phase noise.

Amplifiers (PA) at saturation, which is not the case with the other waveforms.

\section{CONCLUSion}

In this paper, we presented simulated sub- $\mathrm{THz}$ communications impaired by phase noise. We assumed that phase noise is modelled as a Gaussian process. We provided simulations results and compared 11 waveforms using five performance metrics (BER, PAPR, ACPR, SE and EVM). Eventually we discussed the results and then we recommanded to use GMSK or QPSK waveforms in medium to high phase noise impaired system.

\section{ACKNOWLEDGMENT}

The research leading to these results received funding from the French LabEx CominLabs and French administrative region Bretagne within the frame of the project WASSAP.

\section{REFERENCES}

[1] European Communications Office Frequency Information System, "https://efis.cept.org/view/search-general.do", last consulted on 04/01/2020.

[2] T. Nagatsuma, "Advances in terahertz communications accelerated by photonics technologies," in Proc. 24th OptoElectron. Commun. Conf. Int. Conf. Photon. Switching Comput., 2019, pp. 371-379.
[3] S. Bicaïs and J-B. Doré, "Phase Noise Model Selection for Sub-THz Communications", IEEE Global Communications Conference 2019, Dec 2019.

[4] M. Khanzadi, D. Kuylenstiema, A. Panahi et al., "Calculation of the Performance of Communication Systems from Measured Oscillator Phase Noise”, IEEE Trans. Circuits Syst. I Reg. Papers, vol. 61, no. 5, pp. 1553 1565, May 2014.

[5] S. Bicaïs, J.-B. Doré and J.-L. Gonzalez Jimenez, "On the Optimum Demodulation in the Presence of Gaussian Phase Noise", 2018 International Conference on Telecommunications (ICT), 2018, pp. 269-273.

[6] S. Bicaïs and J. -B. Doré, "Design of Digital Communications for Strong Phase Noise Channels," in IEEE Open Journal of Vehicular Technology, vol. 1, pp. 227-243, 2020.

[7] Hillger, P., van Delden, M., Thanthrige, U.S.M. et al. Toward Mobile Integrated Electronic Systems at THz Frequencies. J Infrared Milli Terahz Waves 41, 846-869 (2020). https://doi.org/10.1007/s 10762-020-00699-X

[8] S. Bicaïs and J. -B. Doré, "An Algebraic Framework for Digital Envelope Modulation," 2019 27th European Signal Processing Conference (EUSIPCO), A Coruna, Spain, 2019, pp. 1-5.

[9] K. KrishneGowda, T. Messinger, A. C. Wolf, R. Kraemer, I. Kallfass and J. C. Scheytt, "Towards 100 Gbps Wireless Communication in THz Band with PSSS Modulation: A Promising Hardware in the Loop Experiment", Ubiquitous Wireless Broadband (ICUWB) 2015 IEEE InternationalConference on, pp. 1-5, Oct 2015.

[10] J.-B. Dore, Y. Corre, S. Bicais, J. Palicot, E. Faussurier, D. Ktenas, et al., "Above-90 GHz Spectrum and Single-Carrier Waveform as Enablers for Efficient Tbit/s Wireless Communications", 25th International Conference on Telecommunications (ICT), 2018.

[11] W.D.T. Davies, "System Identification for self-adaptive control". WileyInterscience, 1970 